animals that depend on chemosynthesis. For that to be the case, they must at least provide reductants for long enough to allow chemosynthesis-dependent species to reproduce - more than five years for many vent species ${ }^{13}$ - and be common in space relative to other reducing habitats.

If the large (up to $10 \mathrm{~cm}$ ) vesicomyids on and around the whale bones grow at the high rates measured for hydrothermalvent vesicomyids ${ }^{13}$, the reducing habitat must have been present for at least 3-17 years. Assuming, conservatively, that whale skeletons support chemosynthesis for five years in the deep sea, and that only half of the approximately 1,000 adult grey whales (Eschrichtius robustus) that die each year in an $8 \times 10^{5}-\mathrm{km}^{2}$ area of the north Pacific ${ }^{23}$ sink to, and remain on, the sea floor (also a conservative estimate; D. W. Rice and D. Withrow, personal communication), there would be approximately one reductant-rich skeleton per $300 \mathrm{~km}^{2}$. If these carcasses were randomly distributed over the grey whale range, the average distance between nearest neighbours would be about $9 \mathrm{~km}$ (ref. 24). In fact, deaths and sinking carcasses seem highest along migration routes between Alaskan waters and the Gulf of California ${ }^{2425}$. These rough but probably conservative calculations suggest that whale skeletons may indeed provide persistent and abundant stepping stones for dispersal of deepsea chemosynthetic communities over large areas of the north-east Pacific.

C. R. SMITH H. KUKERT

Hawaii Institute of Geophysics,

School of Ocean and Earth Science and Technology,

University of Hawaii at Manoa,

Honolulu. Hawaii 96822, USA

R. A. WHEATCROFT

P. A. JUMARS

J. W. DEMING

School of Oceanography,

WB-10, University of Washington,

Seattle, Washington 98195, USA

\section{Greenhouse dust}

SIR-Recent concern expressed by the scientific community and by ecologists over anthropogenic 'greenhouse gases', notably $\mathrm{CO}_{2}$, has been attended by a neglect of other factors that contribute to a fluctuating background level of atmospheric (surface) temperature ${ }^{1}$. It is well known that the mean surface temperature can fall by amounts ranging from a few tenths of a degree to a degree or more in extreme cases immediately following a major volcanic eruption, and that the effect can persist for a year or two $\mathrm{o}^{2.3}$. For example, the Tambora eruption of 1815 led to the 'year without summer' and smaller effects are clearly observed in more recent volcanic events, including the Mount St Helens eruptions of 1980.
Whether the surface temperature falls or rises following an injection of volcanic particles, and by how much, is a complex issue depending on, among other factors, the particle size distribution and bulk optical properties ${ }^{4}$.

Another important background effect could result from the accumulation at the Earth of extraterrestrial dust particles interplanetary dust including Brownlee particles (a subset of interplanetary dust particles sampled in the Earth's upper atmosphere) and particles arising from direct encounters with small icy comets. These particle inputs are all highly variable as well as uncertain. The calculations that follow are therefore illustrative, but not definitive. The Brownlee-particle influx, estimated from direct collections of $\sim 10$ - $\mu \mathrm{m}$-sized particle clusters, may be $\sim 10^{16} \mathrm{~g} \mathrm{yr}^{-1}$ (using data in ref. 5), but a submicron component, which is expected to be present, could result in a higher total flux $\sim 10^{11} \mathrm{~g} \mathrm{yr}^{-1}$. With an estimated stratospheric residence time of $\sim 10 \mathrm{yr}$ for the smallest grains we could reasonably expect an average load of $\sim 10^{12} \mathrm{~g}$ of extraterrestrial particulate material, giving a surface density of $\sim 2 \times 10^{-7} \mathrm{~g} \mathrm{~cm}^{-2}$ over the entire Earth.

For the highly porous silicate or organic particles of radii $\sim 10^{-5} \mathrm{~cm}$ expected from comets and micrometeorites, a typical mass extinction coefficient as a result of scattering at visible wavelengths is of the general order of $10^{5} \mathrm{~cm}^{2} \mathrm{~g}^{-1}$ (ref. 6). This is about $10-100$ times the value of the mass absorption coefficient for silicates or organic solids at their infrared absorption peak near the wavelength of $\sim 10 \mu \mathrm{m}$. For a surface density $\sim 2 \times 10^{-7} \mathrm{~g} \mathrm{~cm}^{-2}$ of such particles, which are effectively 50 per cent backscatterers, an effective optical depth for attenuation of visible sunlight available for heating the surface is $\sim 0.5 \times 10^{5} \times 2 \times 10^{-7} \simeq 0.01$, whereas their contribution to heating through infrared absorption will be negligible.

In view of the highly variable nature of the extraterrestrial particle load a typical fluctuation of this effective optical depth will also be of the same order, $\Delta \tau \approx 0.01$. The solar input for heating could thus fluctuate by $\Delta F / F \approx 1-e^{-0.01} \approx 0.01$, which corresponds to a temperature fluctuation $\Delta T / T \approx \Delta F / 4 F \approx 0.25$ per cent. Because temperature fluctuations of about this magnitude are being discussed in connection with the greenhouse problem,

1. Broecker, W.S. Nature 328, 123 (1987).

2. Lamb, H.H. Climate Present, Past and Future Vol. 2 (Methuen, London, 1977).

3. Deepak, A. (ed.) Atmospheric Effects and Potential Climatic Impact of the 1980 Eruptions of Mount St Helen (NASA, CP-2240, 1982)

4. Toon, O. B. \& Pollack, J.B. J appl Meteor. 15, 225 (1976)

5. Brownlee, D.E. in Cosmic Dust (ed. McDonnely, J.A.M Witey, New York, 1978).

6. Wickramasinghe, N.C. Light Scattering Functions for Small Particles (Hilger, London, 1973).

Hoyle, F. \& Wickramasinghe, N.C. Astrophys. Space Sci. $53,523(1978)$.

8. Zhao, M. \& Bada, J.L. Nature 339,463 (1989). we suggest that a proper assessment of the matter must include a consideration of processes of the type discussed above. Small decreases in the background load of extraterrestrial dust would give rise to apparent increases in global temperature. Large increases of this load, which would occur on rare encounters with large comets, could lead to ecodisasters ${ }^{7.8}$.

N.C. WICKRAMASINGHE

F. HOYLE

R. RABILIZIROV

School of Mathematics,

University of Wales, College of Cardiff, Cardiff, CF2 4AG, UK

\section{Cold fusion and brown dwarfs}

SIR-Jones et al. ' claim to have observed neutrons resulting from cold nuclear fusion of deuterons within the palladium metal lattice. They state that observation of naturally occurring ${ }^{3} \mathrm{He}$ in the Earth (again attributed to cold $\mathrm{p}-\mathrm{d}$ fusion occurring in geophysical environment at a rate of $\sim 10^{-24}$ fusions per $\mathrm{d}$ atom per second) and the excess luminosity radiated by Jupiter suggested to them new directions for laboratory studies of cold nuclear fusion.

For instance, the $10^{18} \mathrm{~W}$ radiated by Jupiter could be produced by $\mathrm{p}-\mathrm{d}$ fusions at a rate $\lambda_{\mathrm{f}} \approx 10^{-19}$ fusions $\mathrm{d}^{-1} \mathrm{~s}^{-1}$. Recently, objects intermediate in mass between Jupiter and a typical star, brown dwarfs, have also been attracting attention; some may have been detected ${ }^{2}$. An object about 20 times the mass of Jupiter $\left(20 M_{\mathrm{J}}\right)$ would have a central density $\varrho_{c}$ (which for such objects, with near degeneracy at their cores, scales as $M^{2}$ ) about 400 times larger. An object of $30 M_{\mathrm{J}}$ would have $\varrho_{\mathrm{c}} \sim 900$ times larger, and so on. Their central pressure scales as $M^{10 / 3}$ and would then be much larger than that of Jupiter. So, for a similar $p-d$ composition (which one can reasonably assume for unevolved stellar objects), such objects would have a cold fusion rate $\varrho_{c}^{2}$-times larger (the product of number densities of $p$ and d) than Jupiter. A $20 M$, brown dwarf undergoing $\mathrm{p}-\mathrm{d}$ cold fusion would radiate $\sim 1.6 \times 10^{22} \mathrm{~W}$ or nearly $10^{-4}$ of the solar luminosity. But from their extreme faintness such objects are deduced to emit only $10^{-7}$ solar luminosity or less (even for an estimated $50 M_{1}$ object which should be much brighter, with $6 \times 10^{-13}$ fusions $\left.\mathrm{d}^{-1} \mathrm{~s}^{-1}\right)$. One can reasonably conclude that Jupiter is not getting its luminosity from such reactions which should have made brown dwarfs appear several magnitudes brighter.

C. SivARAM

VENZO DE SABBATA

World Laboratory, Lausanne,

Switzerland

1. Jones, S.E, et al. Nature 338,737-740 (1989)

2. Latham, D.W. et al. Nature 339, 38-40 (1989) 